\title{
Sensibilités métisses et affinités électives
}

Mantuanas et pardas dans le Venezuela du XVIII ${ }^{\mathrm{e}}$ siècle

\section{Frédérique Langue}

\section{(2) OpenEdition \\ Journals}

Édition électronique

URL : http://journals.openedition.org/clio/7424

DOI : $10.4000 /$ clio. 7424

ISSN : 1777-5299

Éditeur

Belin

\section{Édition imprimée}

Date de publication : 1 janvier 2008

Pagination : 75-99

ISBN : 978-2-85816-973-3

ISSN : 1252-7017

Référence électronique

Frédérique Langue, «Sensibilités métisses et affinités électives », Clio. Histoire, femmes et sociétés [En ligne], 27 | 2008, mis en ligne le 05 juin 2010, consulté le 30 avril 2019. URL : http://

journals.openedition.org/clio/7424; DOI : 10.4000/clio.7424

Ce document a été généré automatiquement le 30 avril 2019.

Tous droits réservés 


\title{
Sensibilités métisses et affinités électives
}

\author{
Mantuanas et pardas dans le Venezuela du XVIII ${ }^{\mathrm{e}}$ siècle
}

\author{
Frédérique Langue
}

1 L'écriture de l'histoire, par les choix plus ou moins implicites qu'elle comporte, et l'influence des paradigmes historiographiques qui la sous-tendent, est, par nature, dévoilement et explicitation, construction voire réinvention permanente de son objet. Processus social quant à son élaboration, elle procède également d'un constat, celui d'une impasse première quant à la perception effective des imaginaires du passé. Décrypter dans une perspective différentielle des sources communément plébiscitées par l'histoire sociale et plus particulièrement l'histoire urbaine, dépasser le silence de ces mêmes sources en conférant existence et statut aux paroles tues, à des manifestations d'altérité ou de résistance délicates à " catégoriser » et plus encore lorsque celles-ci relèvent de la sphère plébéienne, a pour vertu principale de mettre en évidence une parole réprimée aussi bien dans un passé révolu que dans un temps présent. Ce sont des langages spécifiques et significatifs, porteurs d'identités elles aussi revendiquées et/ou réprimées, génératrices d'émotions, de solidarités, de compassion, de souffrance, de tension ou de rejet. D'où le rôle qui incombe à l'historien du sensible, celui de prêter un regard attentif aux échos de la rue tels que les restituent les archives, bien distincts de l'échelle prise en compte par les administrateurs de l'empire espagnol pour ce qui est de la réception du même événement ou encore de la vision du monde de ses scribes. Le récit restitue en effet des contextes et des lieux de vulnérabilité et d'incertitude. Il tire de l'oubli les catalyseurs qu'ont été certains événements - fussent-ils de faible intensité et sans pour autant sacrifier au "péché événementialiste » contre lequel Fernand Braudel ne manquait pas de mettre en garde. Il en est de même des situations de violence, explicites ou dissimulées, des modes singuliers ou collectifs d'exister au quotidien, des séquences de vie, des paroles qui se font elles-mêmes événement, toutes situations conflictuelles qui se situent à la confluence du religieux - de la croyance - et du politique, et éveillent incontestablement des échos dans l'imaginaire d'hier et d'aujourd'hui. Tels sont les liens, chaque fois revisités, entre les hommes du passé et ceux du présent. Dès lors, l'espace mouvant de la 
ville coloniale forme intrigue, il se superpose aux discours des élites alors que s'y confrontent de nouvelles formes d'altérité ${ }^{1}$.

2 Discerner les interstices d'où émergent d'autres mécanismes de rationalité, le «non-dit de l'histoire ", ce parti pris en faveur des marges et des zones d'ombre de la discipline, ces émotions et passions qui se meuvent dans la sphère d'un quotidien étranger dans la plupart des cas à celui des élites gouvernantes, est aussi une exploration des désordres apparents qui prévalent dans la société considérée et rencontrent des échos dans l'imaginaire d'aujourd'hui. Cette manière non conforme et inédite de faire de l'histoire a suscité de singulières vocations dans l'historiographie américaniste. Alors que dans les premiers temps de cette tendance historiographique que fut l'histoire des "mentalités ", le Mexique s'était affirmé comme leader continental, avec des études centrées sur le thème du religieux et par conséquent à l'univers de la transgression (histoire du corps, mais surtout, de la famille, tendance qui persiste aujourd'hui encore), d'autres pays, le Venezuela notamment, parvinrent à en préciser les tenants et aboutissants en la reliant presque systématiquement à l'histoire des idées. Le panorama actuel de la recherche américaniste consacre à cet égard l'essor du Brésil, et son inclusion formelle dans le courant de l'histoire des sensibilités, bien que dans une perspective fondée pour l'essentiel sur l'histoire littéraire et l'histoire de l'art, à la différence de l'évolution relevée dans d'autres pays, plus orientés vers la question des représentations ou des problématiques d'histoire sociale ${ }^{2}$.

3 L'histoire des sensibilités s'affirme comme une véritable "caméra subjective » contre la cécité de l'histoire. L'" anthropologie sensorielle", telle que la circonscrit Alain Corbin, propose une lecture autre de l'événement et de sa mise en contexte, allant jusqu'à identifier, en un jeu de correspondances qui impliquent la sensibilité propre de l'historien et donnent lieu à un jeu de miroirs entre passé et présent, de véritables "paysages sensibles $»^{3}$. L'option retenue par Arlette Farge, celle des marges et des frontières de la discipline historique, vise au dépassement de l'« impression fallacieuse d'inéluctabilité de l'histoire » et remet en scène ces « discontinuités » chères à Michel Foucault : celles de ces oubliés du XVIII ${ }^{e}$ siècle et de leur expression au quotidien, via une parole contrainte, voire " consignée dans un ordre d'enfermement » ou pourchassée dans un espace public qui est celui des élites, mais qui, indubitablement, fait sens. Il arrive en effet que des mots, des paroles, des discours voire des rumeurs soient rapportés, en réaction ou à l'origine d'un événement, dans une "brèche » ouverte dans le silence des sources et des consignations officielles. Il y a "rumeur profuse", parole "sur» ou "contre » ce qu'il convient d'appeler la "chose publique». Emergent aussi des "histoires singulières", certes délicates à percevoir, celles que traduisent émotions et sentiments. En d'autres termes, des « sensibilités » sont à l'œuvre. L'injure, même lorsqu'elle repose sur une défense de cet honneur si présent dans le monde hispanique, à tous les niveaux de la société coloniale, se fait ainsi «délit de parole » et fait sens. Communication au sens plein du terme, elle peut influer sur la constitution d'une opinion publique, fût-ce au niveau local, du voisinage, si important lorsque l'honneur des individus ou de leur parentèle est en jeu. La situation ne sera pas différente dans les cas de divorces que nous avons rencontrés, motivés précisément par la violence faite aux femmes et les difficultés de son énonciation. S'y exprime ainsi la violence faite à certaines catégories socio-ethniques et les déviances en résultant dans l'ordre social ou moral ${ }^{4}$. Ce choix de l'indécelable cependant « dicible » conduit de la sorte à la prise en compte de la dimension temporelle, par laquelle un événement n'est jamais toujours le même, est constamment repensé et 
vécu en fonction d'un imaginaire hétérogène et par là même d'une sensibilité sans cesse renouvelés 5 .

4 La dimension temporelle ne peut être dissociée en effet d'une autre problématique qui est celle du statut de l'historien, de ses déterminations dans le champ de l'histoire culturelle, de l'écriture de l'histoire et de la structuration de la mémoire historique, en contrepoint, précisément, aux déterminants idéologiques contemporains, qui ne contribuent souvent qu'à obscurcir la compréhension du présent ${ }^{6}$. Cette sorte de "caméra subjective » que manie l'historien en faisant revivre le passé (l'expression est d'A. Corbin) confère du sens à cet espace controversé qui s'ébauche au fur et à mesure que l'on aborde les sensibilités des exclus, et que ces acteurs oubliés de l'histoire se transforment peu à peu en êtres visibles et identifiables ?. Dans le domaine américaniste, plusieurs publications relativement récentes se sont risquées sur ce terrain, s'attachant à offrir d'autres voies d'interprétation afin de déchiffrer les manifestations et les codes sensibles du passé. Faire sortir de l'ombre les oubliés de l'histoire académique ou officielle, les marginaux, délinquants, femmes de mauvaise vie et autres "pécheurs" d'Amérique espagnole, femmes et hommes du peuple, métis et autres personnes de "couleur cassée " (traduction littérale de color quebrado) conduit en effet à discerner un discours autre, que l'ordre social rejette, en vertu de ces «vices privés et vertus publiques». Mais il est porteur en dernière instance, d'une réelle fluidité des relations sociales, et en tout premier lieu dans le microcosme de la sphère privée (résidences urbaines ou haciendas de cacao dans le cas du Venezuela colonial). Rompant ce silence officialisé y compris dans la sphère du quotidien (el perpetuo silencio), femmes et métis, "classes dangereuses " par excellence dans le Venezuela de la veille de l'Indépendance, font de la rupture des frontières normatives leur apanage. Les uns comme les autres réadaptent en fait et instrumentalisent le modèle initial fondé sur un code de l'honneur on ne peut plus restrictif, revendiquant leur propre "noblesse», déclenchant des passions en retour, réveillant affects et émotions. L'économie des pratiques tend ainsi à déboucher sur une " économie des passions ". $^{8}$.

5 Cette parole en partie sous surveillance - celle des femmes, celle des métis (ses) - l'est également dans l'histoire officielle sur le long terme, alors même qu'elle recèle témoignages et indices révélateurs d'une autre manière d'être au monde. Le genre peut ici constituer une approche complémentaire. L'événement catalyseur et le régime de temporalité qui lui est associé constituent pour l'histoire des sensibilités autant d'approches dans des contextes cependant extrêmement précis, celui des acteurs sociaux de cette configuration et de leur vision du monde. Le "temps de l'événement » nous renvoie par conséquent à un événement vécu sur un registre individuel, à une expérience émotionnelle comme à une suite de réactions qui se situent sur des échelles variées mettant en scène le singulier par rapport au collectif. Les frontières entre les mondes de l'ordre et du désordre, l'inégale appropriation de l'espace urbain par des habitants provenant de deux mondes bien différenciés, contribuent ainsi à la construction de représentations identitaires fondées sur des perceptions éminemment sensibles 9 .

6 L'éphémère, le détail en apparence insignifiant à la première lecture d'un document tiré des archives judiciaires du XVIII ${ }^{e}$ siècle vénézuélien, l'événement « de faible intensité », la rumeur porteuse d'« actualité », l'« indice ", acquièrent ici une importance. Ce qui semble être dérisoire ou dépourvu d'une visibilité riche de sens ne l'est qu'en fonction d'un jugement, à tout le moins d'un choix en vertu duquel on ne peut comprendre ou que l'on est conduit à méconnaitre et même à effacer, qu'il s'agisse de passions oubliées, d'affects 
disparus et indissociables par exemple d'ambiances sonores ou de perceptions auditives enfouis dans un passé par trop lointain et par là même révolu quant à sa (re) transcription et restitution dans l'ordre sensoriel. Une telle lecture de l'environnement s'insère par conséquent dans des processus de construction des identités, aussi bien sur le plan individuel que dans l'ordre collectif, selon le niveau ou l'échelle d'observation d'où se déploie l'analyse des faits considérés. Le fait féminin n'est à cet égard, qu'une "découverte" récente, l'intérêt analytique et non plus seulement descriptif pour l'histoire des femmes ou une approche en terme de genre étant lié par ailleurs à certains grands courants historiographiques, et tout particulièrement à celui des «mentalités » ou des « élites régionales $»^{10}$.

\section{Chimères d'honneur et de couleurs... ${ }^{11}$}

7 Parcourir les archives d'Amérique espagnole à la recherche des acteurs d'un passé retranscrit mais également remodelé par les scribes de l'époque est une rencontre constante et renouvelée avec un modèle culturel hispanique, et plus encore lorsqu'on se penche sur ces révélateurs par excellence que sont, dans le Venezuela du XVIII siècle, les conflits et procès impliquant la gent féminine. Ledit modèle fondé sur la mise en exergue de l'honneur - d'une lignée familiale voire d'un lignage avant d'être celui d'un individu présente d'ailleurs une caractéristique singulière, celle d'être, au Venezuela plus que dans toute autre région d'Amérique espagnole, en évolution et en négociation constante. C'est même la plasticité de ce modèle de comportement, la fluidité de certaines frontières du social qui s'impose au détour des documents, à travers les réticences manifestées dans l'ordre « ethnique » par les différents groupes sociaux. Dans le cas des femmes, une autre déclinaison du principe de l'honneur (honor) est en jeu (honra) ${ }^{12}$, fondée sur des circonstances personnelles qui renvoient à la « publicité » inhérente à tel comportement ( fama) et plus encore lorsque l'on quitte la sphère privée.

8 La prégnance de ce thème - l'honneur des femmes et, par extension, de leur compagnon a pour contrepartie l'extrême discrétion qui prévaut en cas de transgressions commises par les strates supérieures de la société des Indes, et tout particulièrement par l'aristocratie mantuana. L'aristocratie créole du Venezuela colonial est en effet mise hors d'atteinte par un mur de silence - le «silence perpétuel » selon l'expression constamment relevée dans les documents de l'époque - dont les autorités ecclésiastiques sont les garants les plus efficaces. Tel fut le cas de Juan Vicente Bolívar, père du Libérateur, qui faisait preuve d'empressement notoire pour ne pas dire excessif à l'égard des indiennes placées sous sa protection, dans l'ordre spirituel et moral notamment mais non exclusivement (indios de doctrina). Ces personnages d'exception, créoles blancs de noble origine, partagent en effet avec le clergé local et les autorités séculières un pouvoir de fait. Eux-mêmes sont présentés comme des "modèles" pour ce qui est de leur comportement. Ce sont les "pères de familles" mis en valeur dans les Constitutions synodales (1687), recueil de droits et devoirs traditionnellement destiné à combattre les manifestations d'idolâtrie et les manquements à la foi des habitants de la province de Caracas. Ce recueil fondamental pour l'étude de la vie spirituelle du diocèse ${ }^{13}$, dénonce en effet la «multitude prompte à la promiscuité » (multitud promiscual) vivant - à la différence des précédents - sous l'empire des passions, sans retenue aucune, comme le souligna à maintes reprises l'évêque Diez Madroñero. 
Dans les faits, si la dualité de cette réalité sociale s'avère incontournable, il n'en reste pas moins que les strates inférieures de la société d'Amérique espagnole contribuèrent sans la moindre ambiguité à reproduire ce modèle aristocratique et son code de l'honneur, se réappropriant ce modèle avec une force insoupçonnée. Les réclamations émanant des indiennes séduites par le "père de famille » Juan Vicente Bolívar ne conduisirent certes pas à la réparation de l'honneur des intéressées, malgré les protestations de l'évêque Diez Madroñero lors de sa visite pastorale au village (pueblo de indios) de San Mateo ${ }^{14}$. En 1786 cependant, María Teresa Rengifo présenta une réclamation du même ordre devant le Gouverneur capitaine général : le métis (pardo) Sebastián Agudelo l'avait en effet séduite en lui promettant le mariage, puis abandonnée sur les instances de son père Rosalio. Consciente de ses droits, cette demoiselle de bonne conduite (doncella recogida) argumenta que le mulâtre libre, après l'avoir séduite, l'avait abandonnée « déshonorée et ne sachant que faire » alors même que Sebastián n'était " qu'un mulâtre libre »:

Je suis une métisse (parda) libre, honnête et vertueuse. Je suis maintenant déshonorée, et porte l'enfant que m'a valu la promesse de ce Sébastien, qui n'est qu'un mulâtre libre qui n'est en aucune façon d'un rang social supérieur au mien, bien au contraire...

10 À la suite de cette intervention fondée sur l'excellente réputation de l'intéressée, le gouverneur exigea de Rosalío (le distinctif de Don n'étant pas utilisé, on peut donc supposer qu'il s'agit d'un métis à la peau foncée, qui n'a pu se faire passer pour blanc ${ }^{15}$ ) qu'il accorde à son fils l'autorisation de se marier. Les arguments avancés par l'intéressé afin de justifier son refus - María Teresa n'aurait été qu'une "femme des rues », sans distinction aucune, prompte à pratiquer « tous les vices que ce siècle offre à une femme libre... » n'ont pas convaincu le gouverneur qui fut de plus peu édifié par l'image publique et le comportement tout aussi déplorable que le jeune métis présenterait aux yeux du voisinage (thème de la fama mais aussi du pundonor de la femme).

11 Un autre épisode similaire agite la fin du siècle. Une femme noire, María Tomasa Churión, se présente devant les tribunaux en raison de fiançailles rompues. Elle dénonce sans détours Matías Volcán, fils du forgeron Francisco, « qui se considère comme Blanc » mais est marié à une Noire et réclame que les prétendants ne soient pas jugés en fonction de la Pragmatique royale (Real Pragmática) vu qu'ils sont « tous deux mulâtres et n'entrent donc dans aucune des catégories prévues par ce texte $»^{16}$. L'utilisation de ce texte par les gens du peuple, métis, noirs et même, esclaves, montre en fait que la respectabilité sociale reste liée, quelle que soit la catégorie socio-ethnique concernée, à une certaine interprétation du concept d'honneur. Les gens de couleur se montrent même beaucoup plus chatouilleux sur ce point, si l'on considère les réticences constantes des parents lorsque leur rejeton souhaite convoler en justes noces avec un conjoint issu des « castes inférieures ». La référence à ce décret royal (promulgué en 1776 puis 1803), à l'origine une disposition destinée à préserver les "pères de famille » et par conséquent les Blancs, nobles de préférence, d'une alliance "inégale " pour leurs rejetons, est constamment utilisée par les autres groupes sociaux lorsque se présente quelque "inégalité » du conjoint, qu'elle soit « ethnique » ou économique, circonstance de plus en plus fréquente vers la fin de la période coloniale. Inversement, les métis qui se sentent lésés par l'attitude des parents la rejettent, la réservant à d'autres catégories sociales. Dans le même ordre d'idée, María Teresa s'est décrite comme "étant mulâtresse de couleur ", " une femme honnête ", vivant "de son propre travail ", arguments qui motivèrent le Capitaine général et gouverneur Guillelmi à décréter que l'opposition manifestée par le père du futur conjoint était sans fondement ${ }^{17}$. Vers la fin du siècle, les protestations en ce 
sens se multiplient, émanant le plus souvent des femmes, et mettant en avant un honneur qui n'a rien à envier à celui des nobles mantuanos. Il ne s'agit plus seulement de honra, mais bel et bien de honor. Lorsqu'en 1797, Josefa Iriarte se plaint au Gouverneur que «Francisco Antonio Miranda, moreno comme (elle), après avoir célébré leurs fiançailles, prétend désormais en épouser une autre ", elle insiste sur le fait que ce procédé porte atteinte à son « honneur » et qui plus est, aux yeux de tous ${ }^{18}$.

\section{Mantuanas : la rébellion au quotidien}

Pour des raisons liées à la condition de la femme dans la société d'Amérique espagnole, la préservation de l'«honneur» d'un lignage (honor) et de l'intéressée (honra), imposent fréquemment le silence lorsque l'aristocratie est en cause. Ce n'est que lorsque réparation oblige, et que la situation est connue de tous, que certains cas se retrouvent devant le juge. La rumeur peut à elle seule constituer motif à réparation. En 1763, lorsque María Nicolasa Villamil se présente à cet effet devant le vicaire ecclésiastique, elle se dit "orpheline, jeune fille noble, honnête, vertueuse et décente» et se plaint d'un prétendant éconduit et que la rumeur publique lui attribue encore, capitaine de son état, qui la poursuit « en actes et en paroles $»^{19}$.

Des familles mantuanas qui donnèrent le plus de publicité à leurs désaccords conjugaux, les Jerez de Aristiguieta furent sans nul doute les plus connues. Le premier dérapage qui intervint dans cette famille, célèbre pour la distinction voire l'érudition de ses femmes les «neuf muses » - se produisit en 1768. José de Castro y Arraoz adressa une lettre au Gouverneur capitaine général Juan Guillelmi (en date du 28 juillet), se plaignit du " martyr » qu'il endurait du fait du caractère "violent, audacieux et insolent » de son épouse Doña Rosa Aristiguieta. Il reprochait à Doña Rosa d'entretenir des «relations illicites » avec un commerçant basque de Caracas, qui n'était autre que l'ancien facteur de la Compañía Guipuzcoana ${ }^{20}$, Juan Agustín Zuáznavar. Cette initiative ne manquait pas de transgresser la loi du silence communément appliquée par les élites locales en de semblables circonstances. Les faits «scandaleux » reprochés à la mantuana étaient «de notoriété publique » et les affrontements entre époux d'une gravité tout aussi notoire. Doña Rosa aurait ainsi menacé son mari d'un couteau afin de défendre l'un de ses enfants. Se considérant lésé dans son honneur, Castro demanda que son épouse soit assignée à résidence ${ }^{21}$, et son rival expulsé de la province, mais sans pour autant demander le divorce. L'intérêt de ce cas réside dans le fait que Doña Rosa avait refusé de regagner le domicile conjugal. Ce n'était certes pas la première fois que l'esprit frondeur des sœurs Aristiguieta enfreignait les canons de la morale chrétienne. Mais leur condition d'aristocrates leur avait jusqu'alors épargné, et malgré la notoriété des situations relevées, aussi bien la « censure » qui prévalait à d'autres échelons de la société coloniale, que les conséquences de cette absence, ce qui leur conférait une marge appréciable de liberté. Il était ainsi de notoriété publique que l'une des filles de Josefa Blanco fondatrice de cette dynastie de femmes cultivées et libres - mariée à un Iriarte, avait pour habitude de recevoir chez elle et à des heures avancées de la nuit l'Intendant Francisco Saavedra. À tous ces échanges, les esclaves prirent une part active, en faveur de l'un ou de l'autre, contraints de toute évidence par leur maître, et plus solidaires selon les dépositions de leur maîtresse et de ses témoins ${ }^{22}$.

En 1799, ce fut Belén qui, après douze ans de mariage, entama un procès à l'encontre de son époux le colonel Joaquín Pérez Narvarte. Le litige portait sur les modalités 
d'administration de la dote de Belén (plus de 12000 pesos) et l'obtention d'une pension pour elle et ses enfants. Au-delà de ce « divorce » avant la lettre (seule la séparation peut être prononcée), ce sont les termes de ces échanges qui importent. L'avocat de Don Joaquín insinua en effet que Belén avait précédemment accepté les propositions de son époux afin d'éviter " une rupture bruyante » (au vu et au sus de tous), circonstance pour le moins surprenante dans le cas de mantuanos si l'on se souvient du « silence perpétuel » qui entoure leurs actes répréhensifs du point de vue moral. Il dénonça également les "visées peu compatibles avec l'honneur " qui étaient celles de Belén. Celle-ci répliqua non en relativisant ou en justifiant de quelque manière sa conduite mais en se fondant sur son statut, supérieur à celui de son mari, simple Blanc péninsulaire qui abusait par conséquent d'une autorité qui n'était pas la sienne. En 1800, une autre Jerez de Aristiguieta, Josefa María, réclame que lui soit accordée l'administration de ses biens propres, mettant en avant l'abandon du domicile conjugal par son mari, le capitaine Antonio Palacios y Xerez. Les désaccords sont liés ici avec les idées politiques des uns et des autres, la mère de Josefa María professant des idées «républicaines " d'après les documents, et les ayant transmises à ses filles, ce qui semblait inacceptable au capitaine en question. Celui-ci était fermement attaché à l'idéal monarchique et à des années lumières des idéaux d'indépendance qui trouvaient déjà à s'exprimer dans les salons ( tertulias) des mantuanos et allaient culminer dans la « conjuration » de ces derniers (selon le terme consacré par l'historiographie patria) en $1808^{23}$. Le conflit prit fin par une séparation (en 1809), alors qu'une autre des «neuf muses ", María Antonia, tentait ellemême non pas d'obtenir une séparation mais bel et bien de "divorcer» de son époux Bernardo Blanco Strickland, déposant une demande en ce sens auprès des autorités ecclésiastiques. María Antonia avait déjà demandé que lui soit accordé comme lieu de résidence celle de son frère (depósito), en raison des mauvais traitements et des menaces de mort qu'elle avait reçus. De même réclamait-elle de pouvoir administrer elle-même ses biens, en particulier ceux qui lui venaient de son premier mariage. Si l'ensemble de ces annotations contribue à mettre en exergue le caractère indépendant des sœurs Aristiguieta, il n'en reste pas moins qu'elles expriment une tension permanente entre une solution "à l'amiable ", rarement obtenue dans ces cas précis, sans grande publicité au sens premier du terme, et la réparation des affronts et des violences subies par les femmes et connues de tous, autrement dit de leur voisinage ${ }^{24}$.

L'exemple le plus palpable du rôle modélisateur et normatif du concept d'honneur, et de son rôle directeur sur le mode de vie des maisonnées nobles de la province, est sans nul doute celui de cet autre divorce qu'ont entamé dès 1785 le couple mantuano formé par Josefa Lovera et Martín Jerez de Aristiguieta. À l'origine de ce conflit tout d'abord d'ordre civil, se trouvent là aussi les biens dont Josefa Bolívar avait confié - de son vivant l'administration à son gendre. "Dissipation» desdits biens, «mauvaise foi » dans l'administration notamment des biens parafernaux (dont l'administration avait été assurée par le mari), "fraudes ", «accaparement de propriétés» (des haciendas de cacao), "vie dissolue » en compagnie des esclaves de la maisonnée, "ses concubines »" s'ajoutent à une autre accusation beaucoup plus grave : Josefa accuse son mari d'avoir attenté à ses jours, d'avoir « conspiré contre sa vie en diverses occasions, usant de poisons et autre subterfuges au moyen de ses propres esclaves, ses concubines » car

il n'a pas vécu maritalement en ma compagnie, il m'a refusé de quoi vivre et me nourrir, m'a porté préjudice par tous les moyens qui lui venaient à l'esprit et que lui suggéraient la haine implacable qu'il me voue depuis plusieurs années, et l'appétit 
insatiable qui le conduit à s'approprier et ma personne et mes biens, afin de continuer à se vautrer dans le luxe, l'excès et la lascivité... Caracas ce genre de pratiques. Il s'agit ici d'un épisode assez rare de violence, d'où les craintes manifestées par l'intéressée, qui mettent en péril non seulement la vie, l'intégrité physique de Doña Josefa mais également l'honneur d'un lignage mantuano. Caracas sur le plan juridictionnel) rend une décision de «divorce perpétuel » à la demande de Don Martín Xerez de Aristiguieta à l'encontre de Doña Josefa Lovera Otañez y Bolívar sa femme légitime et épouse depuis une trentaine d'années. Les raisons invoquées furent les suivantes, à savoir le caractère " public et notoire » des divers adultères dont chacun avait accusé l'autre. Dans la liste des récriminations formulées par Don Martín afin de défendre son honneur figuraient ainsi deux fugues de Doña Josefa, la prédilection de celle-ci pour les festivités en tout genre, en particulier ces diversions à caractère populaire et indigne de sa classe que l'on nommait saraos, sans compter d'autres activités allant à l'encontre de la morale chrétienne, l'adultère qui faisait qu'elle était « continuellement grosse ». D'où la demande formulée par Don Martín d'enfermement de l'intéressée à l'hospice de Notre Dame de la Charité, à la fois hôpital et lieu de réclusion et de rééducation pour les femmes blanches, surtout les pauvres, afin d'« éviter tout autre scandale ou remise en question de son honneur ", le terme utilisé étant d'ailleurs celui d'« insulte à son honneur ». Quant à la conduite de l'aristocrate, personnage connu pour sa brutalité si ce n'est son tempérament violent comme le souligna son cousin le $\mathrm{D}^{\mathrm{r}} \mathrm{Don}$ Juan Félix de Aristiguieta, prêtre fort respecté à Caracas, elle n'était guère exempte de reproches: concubinages répétés, malversations concernant les biens de Doña Josefa, longue est la liste des écarts commis par ce personnage principal (caballero principal), instigateur d'un procès fort strict et défenseur des bonnes mœurs à l'encontre de sa femme, une noble mais "publique pécheresse », que sa condition «principale " semble avoir aidé dans un premier temps à éluder le contrôle vigilant de son époux ${ }^{26}$.

D'autant que celui-ci s'appuyait sur l'argumentation spécieuse selon laquelle

le délit commis par la femme n'est en aucun cas assimilable à celui du mari ; dans le premier cas, il s'agit en effet d'une personne qui, en raison de sa pudeur naturelle, se doit de préserver son honnêteté, le délit commis en est donc d'autant plus laid, vicieux et criminel.

19 L'infidélité de la femme est cause d'infamie non seulement pour sa personne mais aussi celle de son conjoint, atteint dans son honneur et sa réputation. Pour cette raison, si les époux rompent mutuellement la fidélité qu'ils se sont jurée, un délit ne peut compenser l'autre, l'un peut être considéré plus légèrement, l'autre non... D'où la première décision qui parvint à Caracas le 9 juillet 1791 et prévoyait l'exil de Josefa Lovera au village (pueblo) de Santa Lucía (la famille y possédait des haciendas de cacao) où elle devait purger sa peine de "rétention perpétuelle ", transformée l'année suivante en une obligation de résidence chez de proches parents. Le tribunal ne manqua cependant pas de stigmatiser la conduite dépravée de Don Martín et de lui recommander de suivre plus strictement les dogmes de Sainte Foi. Après avoir effectué les exercices spirituels imposés en de telles circonstances, Josefa vivait désormais de maison en maison, en général dans la paroisse d'Altagracia (lieu de résidence de l'élite mantuana), en compagnie de ses esclaves et de sa famille, tout particulièrement de ses enfants bâtards (hijos espurios). Il continua donc 
d'exiger la réclusion de l'illustre mais en définitive intouchable pécheresse mantuana, décision qu'il obtint en 1796 mais qui, jamais, ne fut appliquée ${ }^{27}$.

Passion et honneur sont, de la même manière que les solidarités féminines et héritages familiaux, les éléments constituant le dossier du divorce qui oppose en 1793 Luis José Loreto de Silva à sa légitime épouse María Josefa Ascanio. Lorsqu'en octobre 1793, l'alcalde (maire) le somme de rejoindre son foyer, l'intéressé s'y refusa, alléguant un honneur entaché et les « désordres » de sa maisonnée, y compris les fugues des esclaves, protégés par leur maîtresse. Réfugiée chez sa tante María Manuela - María Josefa avait toute liberté de choisir son refuge - María Josefa dénonça les «violences » commises par son mari mais également par l'alcalde, lequel avait fait jeter en prison l'une de ses esclaves et... alliées. Se fondant sur son statut d'aristocrate mantuana, elle se plaignit amèrement du fait que cet alcalde avait fait violence aux « privilèges (fueros) d'une femme mariée et de bonne naissance contre laquelle on ne peut prendre de décision de justice et plus encore si la demande n'émane pas du conjoint ». Séparé de sa femme depuis près de quatre ans à la date des événements, Loreto passait pour être un sujet « dissipateur des biens de celle-ci », à l'origine de nombreux désagréments pour sa femme et même d'une atteinte à la vie de celle-ci, sans oublier sa cohabitation avec une employée de maison ${ }^{28}$.

\section{L'honneur et l'identité créole revendiqués ou les métisses vertueuses exemptes « de toute mauvaise race »}

Ainsi se définissent en effet les protagonistes du conflit qui se déroule tout au long de l'année 1787 dans une petite ville de la Capitainerie générale du Venezuela, Carora, qui comptait alors quelque 5000 âmes. Ce conflit révélateur des identités recomposées et d'un système de représentations sociales reconfiguré par ses acteurs apparemment marginaux (les femmes) repose sur une multiplicité de variables et de critères, depuis la couleur jusqu'à l'occupation, en passant par le comportement adopté au vu et au sus de tous, le lieu de résidence (le "pueblo» est facteur de respectabilité), les normes de sociabilité, le regard de l'autre, sans compter l'adaptation de valeurs dominantes, ici celles de l'aristocratie créole.

Le prétexte à l'éclosion de ce conflit est constitué sur le plan juridique par la présence d'un juge dit juez receptor de residencia, dont l'une des fonctions consistait à évaluer périodiquement la gestion des alcaldes et autres autorités administratives, et tout particulièrement lorsque ceux-ci abandonnaient leur charge. Ici se trouve fortement remise en question la manière d'agir et le comportement répréhensible du deuxième alcalde (alcalde mayor de segunda elección) de Carora, accusé par trois sœurs Francisca Rosalia, Antonia Ignacia y María Dionisia Alvarez de Rojas d'avoir porté atteinte à leur honneur pendant la durée de sa charge, soit pendant l'année 1784. Celles-ci estimaient en effet avoir été dépossédées de la catégorie socio-ethnique à laquelle elles disaient appartenir, l'alcalde s'étant refusé à utiliser dans leur cas le «distinctif» de Doña. Réservé à la gent mantuana dans les premières décennies de la vie coloniale, cet usage s'était généralisé par la suite, surtout au cours du XVIII ${ }^{\mathrm{e}}$ siècle. La coutume locale et les pratiques effectives des magistrats et autres juristes, autorités civiles et habitants de la Province : il en vint à concerner toute personne à la peau blanche.

Les trois sœurs reprochent par conséquent à l'alcalde d'avoir 
gravement compromis leur honneur en leur refusant le juste et honorifique traitement de Don qui leur était dû, de par une coutume qui avait force de loi et bénéficiait à toute personne au sang pur (de sangre limpia). et à leur statut social » et à être « indemnisées de la qualification négative de mulâtresses par laquelle on prétendait injustement obscurcir [leur] origine limpide». Bien que l'origine noble - c'est de cela dont il s'agit - des trois femmes ne fut jamais véritablement prouvée, elles prétendaient descendre de familles « de la première noblesse et meilleure distinction de cette ville et de celle de Trujillo ", ce qui n'était en fait le cas que de leur père. Elles évoquèrent « tous les honneurs et prééminences » prévus en de tel cas par les lois de Toro et le traité du juriste Solórzano Pereira, la Política indiana ${ }^{29}$.De telles références peuvent certes surprendre, émanant d'habitantes d'une petite ville n'appartenant pas à l'élite sociale de la province, femmes de surcroît et par conséquent aux possibilités extrêmement inégales d'accès à l'éducation (à moins de se trouver dans l'un des somptueux couvents de Caracas, ou d'entrer au premier Colegio de Niñas Educandas, fondé à la fin du siècle) d'autant que cette possibilité était fréquemment refusée aux gens de couleur et plus encore aux esclaves, que ce genre de connaissances risquaient de rendre "prompts à la révolte " comme l'avait souligné le voyageur Francisco Depons ${ }^{30}$. Toutes trois insistent sur le fait que le préjudice encouru l'est en termes d'honneur (honor) et même de « lignage », sans compter leur honneur (honra, donc plus immédiat, lié au comportement dans la sphère du quotidien et à l'opinion qu'en a le voisinage) qui les distingue des «métis et gens de la plèbe » (pardos y gente plebeya):

en une époque où la pratique et l'usage universel de toute cette province veulent que l'on accorde et même ordonne que soit conféré l'insigne traitement d'honneur à toutes les personnes au sang pur [...], cela équivaut si clairement à reconnaître et à distinguer les personnes blanches des métis et mulâtres que le refuser à quelqu'un revient à dire qu'il s'est mêlé à ces races et à ne pas lui accorder le moindre crédit lorsqu'il est mis en cause...

D'où des tentatives répétées pour «laver cet affront », et « indemniser [leur] lignage de cette infamie ", selon l'expression utilisée par les intéressés pour dénoncer l'attitude de l'alcalde et le préjudice qui en résulte pour elles : « atteinte » (à leur honneur), « mépris », "vocabulaire injurieux ", « insulte de la plus grande gravité », " préjudice», « insulte visant à les vilipender», «malice», « délit», «méchanceté intentionnelle». D’où, également, la nécessité urgente d'y remédier par ce procès, y compris par le biais d'un scandale créé à cette occasion, dans la mesure où la noblesse, et le statut d'exception revendiqués par les trois sœurs sont, par essence, notoires et connus de tous. La réparation doit, par conséquent, être équivalente, d'autant qu'elle est liée au peu de cas qu'aurait fait l'alcalde de ses obligations en matière de justice et des motifs d'ordre personnels qui l'auraient incité à adopter cette attitude.

Considérées comme des "personnes blanches de bonne réputation " par leur entourage, les trois sœurs se virent effectivement confirmer leur statut de femmes blanches par le magistrat Francisco de Olmedilla (Caracas, décisions des 16/X/et 26/XI/1784). L'ambiguïté de la situation et le rôle des pratiques effectives, us et coutumes de la province, firent cependant que des réserves apparurent dans le même document quant à leur origine noble. Il fut insisté cependant sur le risque que comportait pour l'ordre public l'attitude intransigeante de l'alcalde, ce à quoi les intéressées répondirent en ces termes:

Clio. Femmes, Genre, Histoire, 27| 2008 
Lorsqu'on nous déclara nobles (hidalgas), ce fut surtout pour effacer le stigmate de mulâtre que l'on nous attribuait, sans prétention aucune pour ce qui est de notre hidalguía...

Cette revendication quant à l'utilisation du «distinctif » de Don/Doña n'est pas un cas isolé. Dans les dernières décennies du XviII ${ }^{\mathrm{e}}$ siècle, des réclamations similaires sont enregistrées, chaque fois plus nombreuses et argumentées, renforcées qui plus est par la promulgation de l'ordonnance royale de 1795 (Real Cédula de Gracias al sacar) qui permit, moyennant versement d'un droit, un certain nombre d'exemptions, et autorisa ainsi plusieurs pardos enrichis à se «blanchir ». La situation était déjà pour le moins confuse, compte tenu du degré de métissage atteint par la société vénézuélienne du moment et des craintes - de soulèvement et, plus largement, de subversion de l'ordre social manifestées à cet égard par l'administration locale et les élites blanches. Lors de sa visite pastorale dans les années 1770, l'évêque Marti en vint à déconseiller que soient mentionnées les origines ethniques des fidèles dans les livres paroissiaux. Entre les élites aristocratiques blanches et la population de couleur, se mouvait en effet une foule de "petits Blancs» (blancos de orilla), sans fortune ni statut social, mais aussi de pardos enrichis et désireux de conforter leur statut social par une charge ou l'entrée à l'Université réservées aux Blancs. Les arguments présentés par les femmes, blancas de orillas ou pardas, sont à cet égard assez semblables : défense de leur honneur (honor et non pas tant honra) et par conséquent de leur statut social réel ou souhaité. Genre et couleur, ou plus précisément une sorte de "pureté de couleur » similaire à la " pureté de sang " revendiquée par les nobles de la Péninsule ou des Indes se conjuguent ici. Les protestations des hommes ou des membres de leur famille se limitent en revanche aux circonstances entourant leur honneur personnel et font intervenir des accusations d'adultère, prouvé ou non. Autre différence: les femmes et leur entourage prennent davantage l'initiative, dans les différents cas d'honneur bafoué : rapts (consentis ou non), promesses de mariage non tenues, "stupre», "relations illicites» ou violences faites à leur intégrité physique ou morale ${ }^{31}$.

Paradoxalement, mais seulement en apparence, un homme va nous permettre de refermer ce dossier de cas exemplaires, mais significatifs, d'une réalité vécue au quotidien. En 1794, toujours dans la petite ville de Carora, un procès est intenté à l'encontre de Nicolás José Gallardo, pour « délit de concubinage avec María Rosa Suárez sa nièce ». Les circonstances (une parenté de $4^{e}$ degré unit les deux contrevenants) conduit Nicolás Gallardo en prison, après dénonciation par deux voisins (vecinos) déjà en conflit avec l'intéressé pour un terrain que celui-ci possédait à la sortie de la ville. Dans le même temps, rappelons que les aristocrates soucieux de préserver leur patrimoine au fil des générations, ou plus précisément le maintien de celui-ci au sein d'une même famille, en viennent à se marier entre cousins germains, avec la bénédiction des autorités ecclésiastiques $^{32}$. La majorité des procès ouverts pour "mauvaise vie» et autres transgressions ont certes ce point de départ, qui conduit bien souvent à relativiser la nature des accusations portées. Il ne nous appartient pas ici de faire la part des choses. Il est en revanche beaucoup plus significatif pour ce qui est de l'appréhension des sensibilités de l'époque et des représentations métisses circulant dans cet entre-deux que nous évoquions, entre ces deux pôles de la société locale que sont les mantuanos et les esclaves, de revenir sur un document adressé par ce prisonnier de bonne réputation et à la « religiosité notoire » au Gouverneur capitaine général, afin d'assurer une défense dont nous n'avons malheureusement pas pu apprécier les effets, la sentence finale n'apparaissant pas dans les dossiers consultés : 
Comme l'honneur est une passion honorable, qui dépend du bon concept que s'en font les hommes, il n'est pas besoin de recourir à des opuscules pour tomber dans l'erreur. Le vulgaire ignorant, à l'imagination agile et prompt à l'excès, en fera un usage contraire. Porté à la critique, il lui suffira d'une parole pour ruiner le crédit d'un tel. S'ils sont plusieurs à porter une telle accusation, des années de vie juste peuvent être réduites à néant en une heure, transformant son objet en démon car, même chez les hommes les plus sages et soucieux de justifications, domine la croyance que ce que l'on entend à l'encontre de son prochain [...], à savoir des paroles nuisibles, ont quelque fondement, alors qu'elles ne sont qu'un sac de plumes que l'on éparpillerait en l'air ${ }^{33}$.

\section{Quelques mots pour conclure} en découle ont révélé sans nul doute l'intensité mais également la complexité des liens entre représentations et pratiques en cette fin du XVIII ${ }^{\mathrm{e}}$ siècle. Malgré la surdétermination des situations et des lieux décrits, les plaintes déposées par les femmes se prêtent à différents axes de lectures, non sans échos dans la vie d'aujourd'hui. La parole individuelle, sa participation, adaptation ou confrontation au conflit créé ou juridiquement assumé, acquiert une fonction qui lui est propre, dans un monde d'Ancien Régime ou les «états » et corporations diverses gouvernent les esprits. Elle remet en question, du moins celle des femmes évoquées ici, l'autorité de dire et de décider - celle des juges et des prêtres - sur un point bien précis : celui de la violence quotidienne vécue aussi bien par les métisses et plébéiennes que par les femmes de l'aristocratie. En ces dernières décennies du XVIII ${ }^{e}$ siècle vénézuélien, la parole des hommes devient sujette à caution, qu'ils soient époux, séducteurs, magistrats et ministres du culte. Les représentations mentales qui orientent le rapport des contemporains au monde, le rapport normatif qui régit les relations de genre se déclinent en des univers d'interactions, de la maisonnée à l'espace public, au « voisinage », sans compter des lieux de travail ou de réunion et discussion (tertulias) que nous n'avons pas détaillés ici. Comme le processus d'Indépendance le montrera, ce savoir social et ces représentations ne sont cependant pas a-historiques, les normes qu'elles régissent en sont infléchies, reconsidérées, reformulées constamment. L'appréhension des écarts de conduite des femmes, telle qu'elle est pratiquée par le monde des religieux, est elle-même fluctuante, et en corrélation avec les transformations du monde laïc, fait de concessions et d'adaptations à la différence d'une justice séculière qui ne cherche nullement à « rédimer » mais à châtier les contrevenants ${ }^{34}$.

Le langage formalisé par les femmes, mantuanas ou métisses revendiquant leur "pureté de couleur ", ce nouveau pouvoir de dire en des situations inédites, est partie intégrante de cette tension entre le système normatif hispanique fondé sur un code restrictif de l'honneur, et les accommodements qui en sont faits. Ces stratégies de contournement, qui s'appuient sur les mutations des codes juridiques conduisent à une réappropriation de ces derniers, comme le montre l'utilisation de la Pragmatique de 1776 non pas tant par les « classes » auxquelles elle était destinée - les élites blanches - mais par un monde métis constitutif des futures « classes moyennes » criollas que Manuel Pérez Vila distinguait déjà au début $\mathrm{du} \mathrm{xIX}^{\mathrm{e}}$ siècle vénézuélien ${ }^{35}$. À la veille de l'Indépendance, la société de la Province de Caracas répond en ce sens à l'observation faite par L.Capdevila et D. Godineau, selon laquelle « toute mutation de la société s'accompagne d'un ajustement 
du genre, c'est-à-dire d'un polissage des stéréotypes du masculin/féminin, d'une variation des identités sexuelles, d'un changement dans les relations hommes/femmes, d'une adaptation des imaginaires sociaux ». Le rôle déterminant des femmes dans ce chamboulement des pratiques va bien au delà d'une reformulation, certes essentielle, mais au quotidien, de leur statut. Plus que leurs pairs mantuanos, présents dans les instances de décision locales et par conséquent de pouvoir (Cabildo, Consulat de commerce), les mantuanas ont joué à cet égard un rôle décisif de médiatrices culturelles. Ce modèle de comportement et de pratiques a été repris, choisi, par ces pardas vertueuses se voulant elles aussi nobles, dans un espace et en des lieux marqués du sceau du quotidien, induisant par conséquent un métissage des pratiques et des représentations. La «culture du peuple» évoquée par N. Zemon Davis trouve ici sa raison d'être, si l'on considère les attitudes, les affinités, les discours voire les connaissances adoptées par des catégories sociales «subalternes". Les mantuanas ont ainsi participé pleinement à la construction de la nation vénézuélienne, d'un côté comme de l'autre, comme le montre l'exemple certes romancé mais pas moins édifiant de María Antonia Bolívar, prenant fait et cause pour la défense... des principes monarchiques et non pour la cause de l'« émancipation » et encore moins de la République telles que les défendaient d'illustres mantuanos dont le plus célèbre fut son frère le Libertador ${ }^{36}$.

\section{BIBLIOGRAPHIE}

ALBORNOZ VÁsQUEz María Eugenia, 2004, « La Injuria de Palabra en Santiago de Chile, 1672-1822 », Nuevo Mundo Mundos Nuevos, 4 : http://nuevomundo.revues.org/document240.html

ALVAREZ DE LOVERA María, 1994, La mujer en la Colonia. Situación social y jurídica, Caracas, Fondo Editorial Tropykos/FACES-UCV.

AMODIO Emanuele, 1995, « Vicios privados y públicas virtudes. Itinerarios del Eros ilustrado en los campos de lo público y de lo privado ", in Lo público y lo privado. Redefinición de los ámbitos del Estado $y$ de la sociedad, Caracas, Fundación Manuel Garciá Pelayo.

BENSA Alban, 2006, La fin de l'exotisme. Essais d'anthropologie critique, Toulouse, Anacharsis.

BENSA Alban, FASSIN, Éric, 2002, « Les sciences sociales face à l'événement », Terrain, 38, p. 5-20.

BRAUDEL Fernand, 1969, Ecrits sur L'histoire, Paris, Flammarion.

CAPDEVILA Luc, CASSAGNES Sophie, COCAUD Martine, et alii, 2003, Le genre face aux mutations. Masculin et féminin, du moyen Âge à nos jours, Rennes, PUR.

CAULFIELD Sueann, 2001, "The History of Gender in the Historiography of Latin America", HAHR, $81,3$.

CERRUTTI Simona, 1995, « La construction des catégories sociales », in Passés recomposés. Champs et chantiers de l'histoire, Paris, Autrement, $\mathrm{n}^{\circ}$ 150-151, p. 224-242.

CORBIN Alain, 1994, Paysage sonore et culture sensible dans les campagnes au XIX siècle, Paris, Albin Michel. 
-, 2000, Historien du sensible. Entretiens avec Gilles Heuré, Paris, La Découverte.

DÁVILA Dora, 1994, « Se tiraban fuertemente al honor. La separación de dos aristócratas a finales del siglo XVIII venezolano », in E. PINO ITURRIETA (dir.), Quimeras de amor..., p. 67 sq.

FARGE Arlette, 1992, Dire et mal dire. L'opinion publique au XVIII ${ }^{e}$ siècle, Paris, Seuil.

-, 1994, Le cours ordinaire des choses dans la cité du XVIII siècle, Paris, Seuil.

—, 1997, Des lieux pour l'histoire, Paris, Seuil.

-, 2002, « Penser et définir l'événement en histoire », Terrain, 38, p. 69-78.

FOUCAULT Michel, 1994, Dits et écrits, t. II, Paris, Gallimard.

FUENTES BAJO María Dolores, 1992, « Familia, matrimonio y poder en la Caracas colonial : el caso de los Jerez Aristiguieta, 1786-1809 ", in Europa y América. Cinco siglos de intercambios, vol. I, Sevilla, AHILA/Junta de Andalucía, p. 371-389.

GARCíA MALDONAdo Ana Lucina (dir.), TROCONIS DE VERACOECHEA Ermila (coord.), 1995, La mujer en la historia de Venezuela, vol. I., Caracas, Asociación Civil La Mujer y el V Centenario de América y Venezuela.

GINSBURG Carlo, 1989, « Traces. Racines d'un paradigme indiciaire », in Mythes, Emblèmes, Traces, Morphologie et Histoire, Paris, Flammarion.

GÓMEZ Alejandro E., 2005, « Las revoluciones blanqueadoras : elites mulatas haitianas y « pardos beneméritos » venezolanos, y su aspiración a la igualdad, 1789-1812 », Nuevo Mundo Mundos Nuevos, 5 : http://nuevomundo.revues.org/document868.html

HABERMAS Jürgen, 1986, L'espace public, archéologie de la publicité comme dimension constructive de la société bourgeoise, Paris, Payot.

KellogG Susan, 2000, "Depicting Mestizaje : Gendered Images of Ethnorace in Colonial Mexican Texts", Journal of Women's History, 2,3. PAGES

KONETZKE Richard, 1975, Colección de documentos para la historia de la formación social de Hispanoamérica 1493-1810, vol. III, tome 1, Madrid, CSIC.

LADERA DE DIEZ Elizabeth, 1990, Contribución al estudio de la « aristocracia territorial » en Venezuela colonial. La familia Xerez de Aristiguieta siglo XVIII, Caracas, Academia Nacional de la Historia (Coll. Fuentes para la Historia Colonial de Venezuela $n^{\circ} 209$ ).

LANGUE Frédérique, 1994a, « L'histoire fragmentée et les pécheurs vertueux. L'histoire des mentalités au Mexique, bilan historiographique », Cahiers d'Amérique Latine, 17, p. 157-162.

-, 1994b, "La historia de las mentalidades y la América colonial (América española, Brasil). Selección bibliográfica", REDIAL. Revista europea de información y documentación sobre América Latina, 4, p. 77-118.

-, 1995, «Les identités fractales : honneur et couleur dans la société vénézuélienne du XVIII ${ }^{\mathrm{e}}$ siècle ", Caravelle, 65, p. 23-37.

-, 1996, "El pensamiento venezolano de la emancipación y la conjuración de los mantuanos", III Congrès Latino-américain de l'Université de Varsovie, 16-18 juin 1995, Memorias, t. 2, Varsovie, p. 97-105.

-, 1997, «La pardocratie ou l'itinéraire d'une "classe dangereuse" dans le Venezuela des XVIII et XIX ${ }^{\mathrm{e}}$ siècles ", Caravelle, 67, p. 57-72. 
-, 1998, "La historia de las mentalidades y el redescubrimiento de las Américas", Revista Actualidades (Centro de Estudios Latinoamericanos Rómulo Gallegos), 7, p. 7-21.

,- 1999 , « Le cercle des alliances. Stratégies d'honneur et de fortune des aristocrates vénézuéliens au XVIII ${ }^{\mathrm{e}}$ siècle », Annales, 54, 2, p. 453-480.

-, 2000, Aristócratas, honor y subversión en la Venezuela del siglo XVIII, Caracas, Academia Nacional de la Historia (coll. Fuentes para la Historia colonial de Venezuela ${ }^{\circ} 252$ ).

-, 2001, « Historiografia colonial de Venezuela, pautas, circunstancias y una pregunta : ¿También se fue la historiografía de la Colonia detrás del caballo de Bolívar ? », Revista de Indias, 222,

p. $247-265$

-, 2004, «Silencio, honor y desgarramiento ; Familias mantuans del siglo XVIII", in Dora DÁvILA (dir.), Historia, género y familia en Iberoamérica (siglos XVI al XX), Caracas, Fundación Konrad Adenauer-UCAB-Instituto de Investigaciones Históricas, p. 103-126.

-, 2006, «L'histoire des sensibilités et l'Amérique latine. Une autre manière d'écrire l'histoire au Venezuela... et ailleurs ", Caravelle, 86, p. 13-30.

-, 2006, « L'histoire des sensibilités. Non-dit, mal dire ou envers de l'histoire ? Regards croisés France-Amérique latine », Nuevo Mundo Mundos Nuevos, 6 : http://nuevomundo.revues.org/ document2031.html

LEAL Ildefonso, 1995, « La educación de la mujer en la época colonial venezolana », in García MALDONAdo, Ana lucina (dir.), troconis de VERACOECHEA, Ermila (coord.), La mujer en la historia de Venezuela, Caracas, Asociación Civil La Mujer y el V Centenario de América y Venezuela, vol. I, chap. VI.

LOVERA REYES Elina, 1995, « Las mujeres y la Iglesia en los tiempos coloniales », in García maldonado, Ana lucina (dir.), troconis de veracoechea, Ermila (coord.), La mujer en la historia de Venezuela, Caracas, Asociación Civil La Mujer y el V Centenario de América y Venezuela, vol. I, chap. VII.

PÉREZ VILA Manuel, 1986, "El artesanado. La formación de una clase media propiamente americana (1500-1800) », Boletín de la Academia Nacional de la Historia, 69, 274, p. 325-344.

PINO ITURRIETA Elías, 1992, Contra lujuria, castidad. Historias de pecados en el siglo XVIII venezolano, Caracas, Alfadil.

-, 1994, Quimeras de amor, honor y pecado en el siglo XVIII venezolano, Caracas, Planeta.

QUINTERO Inés, 2003, La criolla principal. María Antonia Bolívar, hermana del Libertador, Caracas, Fundación Bigott.

-, 2006, « "Los nobles de Caracas”. Discurso de incorporación como individuo de número de la Academia Nacional de la Historia (Caracas) », Boletín de la Academia Nacional de la Historia, LXXXIX, 353, disponible sur : http://www.anhvenezuela.org/

RICœUR Paul, 2000, La mémoire, l'histoire, l'oubli, Paris, Seuil.

SOCoLow Susan, 2005, "Colonial Gender History”, LARR, 40, 3.pages

SOLÓRZANO Katty, 1998, Se hizo seña : medición y percepción del tiempo en el siglo XVIII venezolano, Caracas, Planeta.

TROCONIS DE VERACOECHEA Ermila, 1990, Indias, mantuanas y primeras damas, Caracas, Alfadil/

Trópicos/Academia Nacional de la Historia. 
ZEMON DAVIS Natalie, 1975, Society and Culture in Early Modern France, Stanford, Stanford University Press.

\section{NOTES}

1. Habermas 1986 ; Farge 1992 : 13-14 et 1997 : 149 ; Langue 1994 a \& b, 1998 ; Braudel1969.

2. Un état des lieux de cette jeune histoire des sensibilités dans ses rapports avec l'écriture de l'histoire dans le numéro spécial coordonné par nous, «L'histoire des sensibilités et l'Amérique latine », Caravelle, 86, 2006. Pour le Venezuela, voir les travaux d'Elías Pino Iturrieta.

3. Corbin 1994, 2000.

4. Albornoz Vásquez 2004.

5. Farge 1992, 1997 ; Foucault 1994 : 150 sq ; Solórzano 1998.

6. Langue 1995, $2006 a$.

7. Ricœur 2000 : 449 sq ; Langue 2006b.

8. Nous empruntons cette expression à Amodio 1995 : 169 sq ; Langue 2000 ; Gómez 2005 ; Bensa $2006: 237$.

9. Ginsburg 1989 ; Bensa et Fassin 2002 ; Farge 1994, 2002 ; Kellogg 2000.

10. Langue 1995; Troconis de Veracoechea 1990; García Maldonado (dir.), Troconis de Veracoechea (coord.) 1995 ; Alvarez de Lovera 1994 ; Langue 2001 ; pour le monde anglo-saxon, voir Socolow 2005 ; et le bilan dressé par Caulfield 2001.

11. En référence à la première acception du terme (peut également avoir le sens de querelle) voir l'ouvrage coordonné par Elías Pino Iturrietaen 1994 (Frédérique Langue, Dora Dávila, Rafael Strauss, Luis Felipe Pellicer, Pedro Enrique Calzadilla, Inés Quintero).

12. À la différence de honor, lié ici à la noblesse d'un lignage, la honra renvoie à des circonstances personnelles qui sont celles d'un individu et plus particulièrement d'une femme, nuance que la traduction française ne peut restituer, les deux termes étant traduits par « honneur ». Son sens est donc proche de la «bonne réputation ». Aujourd'hui encore le Diccionario de la Real Academia Española (RAE, ed. 2001) insiste sur ce sens : dignité/bonne opinion, réputation acquises par la vertu et le mérite/l'estime que l'on éprouve pour des personnes présentant ces qualités/pudeur, honnêteté, réserve et modestie chez les femmes.

13. Langue 2004.

14. Pour une mise en perspective du thème des Constitutions synodales et des applications concrètes de ce texte, cf. Pino Iturrieta 1992.

15. Rosalío, à l'instar de nombreux pardos enrichis et en général de couleur claire, n'hésita pas à s'inventer une généalogie qui n'avait rien à envier à celle des aristocrates et de leurs ancêtres «vieux chrétiens » dépourvus comme chacun sait du stigmate plébéien de l'esclavage et du mélange racial. María Teresa et les témoins qui avaient déposé en sa faveur n'auraient en effet été que « des gens très inférieurs et inégaux, élevés sans la décence et retenue dans laquelle ses propres fils avaient été éduqués, mais aussi ses ascendants, ce qui était de notoriété publique... ». Leur nom et qualité renvoyaient à l'« esclavage ", à la «servitude ", et aux "gens de mauvaise vie».

16. La Pragmática Sanción sobre matrimonios desiguales fut promulguée en 1776 en Espagne et étendue aux Indes en 1778. Reproduite dans Konetzke 1975.

17. Langue 1995, pour la perception du pardo à l'époque coloniale; Konetzke 1975. Archives de l'Académie Nationale d'Histoire, Caracas (ANH), Civiles, 1801.

18. ANH, Civiles, 1797.

19. Archives de l'Archevêché de Caracas (AAC), Matrimoniales, 371.

20. Assurait le commerce du cacao entre le Venezuela et l'Espagne. 
21. Depósito : peut intervenir dans des institutions spécialisées, de type carcéral, mais pour les femmes de l'aristocratie, cette condamnation revient à être enfermées chez elles ou dans quelque propriété quelque peu éloignée du lieu du délit.

22. Archives Générales des Indes (AGI), Caracas, 91. Fuentes Bajo, 1992.

23. Langue 1996.

24. Ladera de Diez 1990.

25. ANH, Civiles, 1785 : Josefa Lovera au gouverneur, Caracas, 14/IV/1785.

26. Dávila $1994: 67$.

27. Dávila 1994 : 76.

28. Archives Générales de la Nation (Caracas, AGN) Disensos y Matrimonios, LXX.

29. Lois de Toro : corpus juridique promulgué sous les Rois catholiques à l'occasion des Cortes de Toro (1505). La Política indiana, recueil de droit des Indes (derecho indiano) du juriste Juan de Solórzano Pereira, fut publiée à Madrid en 1647.

30. Langue 1995 ; ANH Criminales : Francisca Rosalia, Antonia Ignacia et Maria Dionisia Alvarez de Rojas au juge, Carora, 7/IX/1878; Leal 1995.

31. Langue 2000 ; et un résumé de la situation et des inquiétudes des mantuanos dans Quintero 2006.

32. Langue 1999.

33. ANH, Civiles, 1794 : Nicolás José Gallardo al Gobernador, Carora, 22/III/1785.

34. Cerrutti 1995.

35. Pérez Vila 1986.

36. Capdevila, Cassagnes, Cocaud, Godineau (et al.) 2003 (conclusion); Zemon Davis1975; Quintero 2003.

\section{RÉSUMÉS}

Le Venezuela de la fin de la période coloniale se distingue sur bien des points des autres régions d'Amérique espagnole : une prospérité économique flatteuse fondée sur le commerce du cacao et que confirment les réformes bourboniennes de la fin du siècle; une configuration sociale contrastée mais extrêmement fluide et une aristocratie créole, les mantuanos, dont le discours et les généalogies sociales sont repris à leur compte par les autres groupes sociaux émergents, notamment par le monde des pardos (métis); mais également l'une des révolutions d'Indépendance les plus violentes du continent. Les sensibilités métisses vont de pair avec une mémoire partagée, au quotidien ou lors d'événements bien particuliers tels que visitas ou procès. D'autant qu'un autre élément contribue à faire de cette société métisse une société en mouvement : les femmes, quel que soit leur statut social ou ethnique. Transgressions des normes sociales et morales par les aristocrates mantuanas, revendications des pardas se voulant nobles et défendant leur "honneur »: se situant dans la perspective de l'histoire des sensibilités, cette étude tentera de faire le point sur ce métissage des représentations, ainsi que sur la subversion du modèle culturel hispanique qui en découle dans les pratiques effectives.

At the end of the colonial period, Venezuela is quite different from any other regions of Spanish America: first an economic prosperity which benefits from the cacao trade and strengthened by the Bourbon reforms at the very end of the century; a very contrasted but fluid social configuration; a creole aristocracy, the mantuanos, whose words and social genealogies are 
retrieved by other emergent social groups, such as the pardos (mestizos, half-caste); one of the most violent revolutions of Independence in the mainland. Hybrid sensibilities emerged along with a shared memory at the everyday level or at specific events such as visitas or trials. In this half-caste society, women, whatever their social or ethnic status, introduced another elements of mobility. The aristocratic mantuanas broke and defied social norms, the half-caste pardas claimed consideration as noblewomen and constantly defended their honour. From the point of view of the history of sensibilities, this study aims to evaluate the miscegenation of social representations as well as the effective subversion of initially Spanish cultural models.

\section{INDEX}

Mots-clés : aristocratie, genre, métissage, représentations, sensibilités (histoire des)

Keywords : aristocracy, gender, miscegenation, representations, sensibility (history of), Venezuela

Index géographique : Venezuela

\section{AUTEUR}

\section{FRÉDÉRIQUE LANGUE}

Chargée de recherche au CNRS habilitée à diriger des recherches (CERMA-MASCIPO, EHESS), est spécialiste d'histoire sociale et des sensibilités de l'Amérique latine dans le long terme, y compris du temps présent (Venezuela). A été professeur des universités vénézuéliennes, et professeur invitée dans diverses institutions latino-américaines. Rédactrice en chef de la revue électronique Nuevo Mundo Mundos Nuevos. Auteur notamment de Mines, terres et sociétés à Zacatecas (Mexique) de la fin du XVII siècle à l'Indépendance, Paris, Publications de la Sorbonne, 1992 ; Aristócratas, honor y subversión en la Venezuela del siglo xviii, Caracas, Academia Nacional de la Historia, 2000 ; avec Sandra Pesavento, de Representações e sensibilidades nas Américas e o Caribe (s. xvi-xxi), Porto Alegre, Editorial da Universidade Federal do Rio Grande do Sul, 2007. 